\title{
Estudio epidemiológico de la Obesidad y el Sobrepeso en una poblacion pediatrica de medio rural de Castilla-La Mancha
}

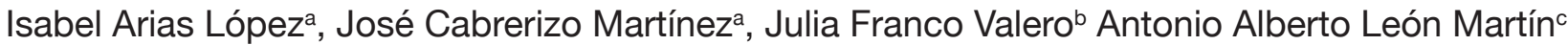

\begin{abstract}
a Pediatra del Centro de Salud de Ciudad Real II.

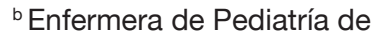
Centro de salud de Calzada de Calatrava (Ciudad Real).

c Técnico de Salud Pública. Unidad Docente de la Gerencia de Atención Primaria de Ciudad Real.
\end{abstract}

Correspondencia: Isabel Arias López. Plaza Mariana Pineda $\mathrm{n}^{\circ} 1,8^{\circ} \mathrm{B}$, Puertollano, (Ciudad Real). Telf.: 676353908 / 926411359, e-mail: _iarias@sescam.jccm.es

Recibido el 19 de marzo de 2008.

Aceptado para su publicación el 13 de mayo de 2008.

\begin{abstract}
RESUMEN
Objetivos. Conocer la prevalencia y la evolución de la obesidad y el sobrepeso en la población pediátrica de nuestro medio de trabajo y si factores como la edad, sexo o hipertensión arterial están asociados a la obesidad/sobrepeso.

Diseño y participantes. Se realizó un estudio retrospectivo de seguimiento de una cohorte de todos los niños nacidos en 1992 en la Zona Básica de Salud de Calzada de Calatrava $(n=92)$. Las fuentes utilizadas fueron las historias clínicas, recogiéndose las siguientes mediciones: peso, talla e IMC de cada paciente en la revisiones correspondientes a recién nacido, 2 años, 6-7 años, 10-11 años y 1415 años, más tensión arterial de las dos últimas revisiones.

Resultados. La prevalencia de Obesidad en nuestra población a los 14 años fue de 9,8\% según criterios de Cole $^{1}$ et al y de 18,3 \% según criterio de Hernández ${ }^{2}$ et al. Respecto a la alteración en $1^{a}$ toma de tensión arterial durante la revisión de los 11 y 14 años, encontramos una relación estadísticamente significativa entre padecer hipertensión arterial y obesidad a los 14 años.

Conclusiones. Con nuestro estudio hemos comprobado que en nuestra población se está produciendo un aumento alarmante de obesidad y sobrepeso, al igual que se refleja en las publicaciones consultadas. La condición de obesidad infantil puede favorecer la presencia de otros factores de riesgo cardiovascular como la hipertensión. Creemos que la obesidad y el sobrepeso son un problema real e instaurado en la población española.

Palabras clave. Obesidad, Niño.
\end{abstract}

\section{ABSTRACT}

Epidemiological study of obesity and excess weight in a pediatric population in a rural setting in Castilla-La Mancha

Objectives. To determine the prevalence and trends in obesity and excess weight in the pediatric population in our work setting and whether factors such as age, sex or blood pressure are associated with obesity/excess weight.

Design and participants. A retrospective follow up study was carried out on a cohort of all the children born in 1992 in the Basic Health Area of Calzada de Calatrava $(n=92)$. The sources used were clinical records, and the following measurements were recorded: weight, height and BMI for each patient in the check ups carried out at birth, 2 years, 6-7 years, 10-11 years and 14-15 years, and blood pressure in the last two check ups.

Results. The prevalence of obesity at 14 years old in our population was around $9.8 \%$ according to criteria of $\mathrm{Cole}^{1}$ et al and $18.3 \%$ according to the criteria of Hernandez ${ }^{2}$ et al. With regards the alteration in blood pressure at the first measurements, during the 11-year and 14-year check ups, we found a statistically significant association between high blood pressure and obesity at 14 years old.

Conclusions. With our study we have shown there to be an alarming increase in obesity and excess weight in our population, in accordance with the literature consulted. Childhood obesity can also favour other cardiovascular risk factors such as high blood pressure. We consider that obesity and excess weight are a real and established problem in the Spanish population.

Key words. Obesity, Child.

Los resultados de este estudio han sido presentados previamente en modalidad póster en el XXI congreso nacional de Pediatría Extrahospitalaria y Atención Primaria, celebrado en Barcelona del 11 al 13 de Octubre de 2007.

\section{INTRODUCCIÓN}

La Obesidad se define como un exceso de grasa corporal. La obesidad es una enfermedad crónica multifactorial, principalmente debida a factores ambientales y genéticos, que determinan un desequilibrio entre la ingesta y el gasto energético.

Debido a que la medición directa de la masa grasa es muy difícil en la práctica clínica, por ello se aceptan otros métodos indirectos de medición como el índice de masa 
corporal (IMC), que es el parámetro antropométrico mejor correlacionado con la grasa corporal. Se define obesidad y sobrepeso a los valores de IMC igual o mayores a los Percentiles 97 y 90 , para su edad y sexo correspondiente, de las tablas de referencia españolas de Hernández ${ }^{2}$ y cols. En el caso de usar las referencias de Cole $^{1}$ et al son los valores de IMC > 30 (percentil 95) y 25 (percentil 85), respectivamente, a los 18 años extrapolados a las edades y sexo correspondientes.

Cualquier incremento de más de dos unidades de IMC anual identifica un rápido incremento de la grasa corporal en niños y adolescentes, debiéndonos poner en alerta ante el posible inicio de una obesidad.

Hay otras mediciones como el pliegue tricipital ${ }^{3}$, que complementaria aún más el diagnostico de obesidad junto a unas tablas de referencia de IMC adecuadas. Debido a que, como hemos explicado previamente, no existe un acuerdo consensuado respecto al punto de corte del IMC para definir la obesidad y el sobrepeso, en nuestro trabajo hemos utilizado los valores de refe- rencia, según edad, sexo y talla, mas utilizados a nivel nacional: los publicados por la fundación Orbegozo 1998 y 2004 y los puntos de corte estandarizados internacionales de IMC de Cole et al. De todos modos, este tema esta aún muy debatido y necesita mejorar para poder hacer comparaciones más exactas de los distintos trabajos.

La obesidad se puede considerar una pandemia pues está aumentando su prevalencia aceleradamente en los últimos años, principalmente en los países desarrollados. En las publicaciones consultadas la prevalencia de obesidad y sobrepeso en niños en España ha variado mucho en los últimos años. En el estudio Paidos ${ }^{4}$ (1984) la prevalencia de obesidad infantil fue del 4,9\% en ambos sexos entre los 6 y los 12 años. Posteriormente, en el estudio enKid ${ }^{5}$, realizado entre los años 1998 a 2000, fue de 13,9\% según el Percentil 97 de Hernández y cols y la del sobrepeso de $12,4 \%$, observándose mayor prevalencia en varones que en mujeres y en edades de 6 a 13 años. En comparación con

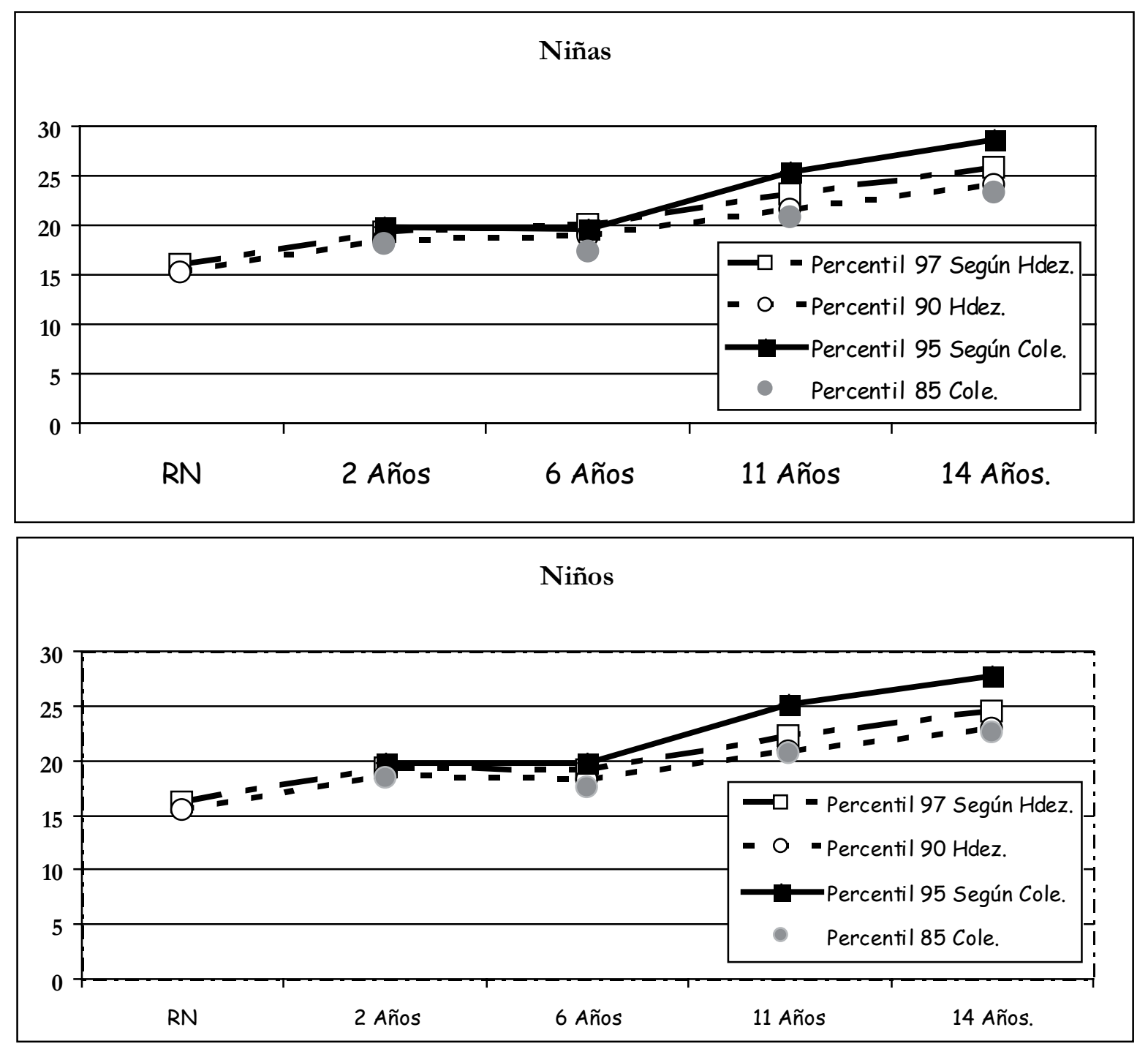

Figura 1. Puntos de corte de obesidad y sobrepeso según Hernández y Cole en niñas y niños. 


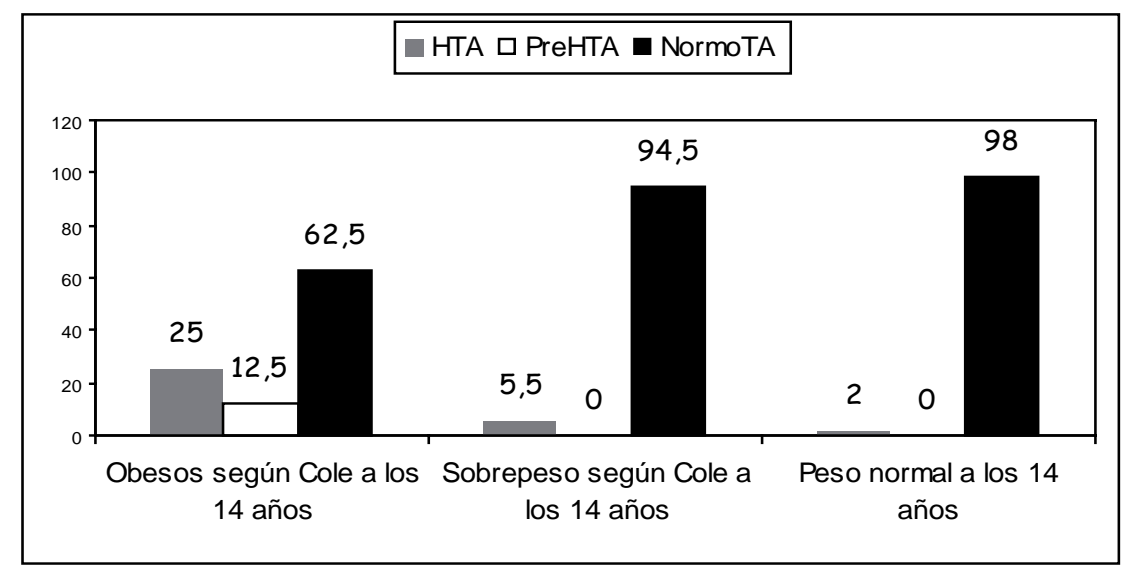

Figura 2. Proporción de hipertensos entre los pacientes de la muestra a los 14 años.

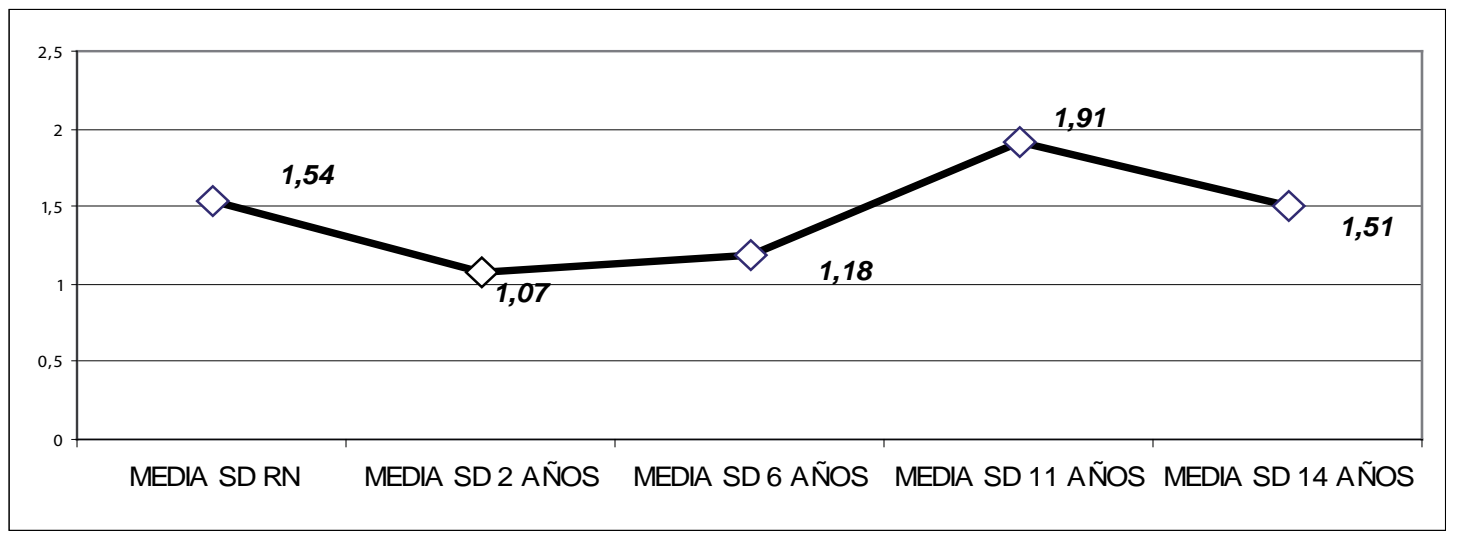

Figura 3. Medias de las desviaciones típicas (SD) del IMC de los sujetos de la muestra sin normopeso.

Estados unidos, la obesidad es superior en España en hombres en edades entre 2 a 8 años y de 12 a 14 , y en mujeres de 2 a 5 años. En el estudio Avena ${ }^{6}$, realizado en 2001-02 en adolescentes españoles, la prevalencia de sobrepeso ascendió a $20 \%$ en hombres y $16 \%$ en mujeres. Según el estudio Internacional Obesity Task Force (IOTF) de 2004, España tendría la $4^{a}$ prevalencia más alta de Europa.

Este aumento también se ha detectado en Estados Unidos, anunciándose en el estudio $\mathrm{NHANES}^{7}$ (1984 a 1993) incrementos en las cifras de obesidad entre los 6 y 11 años en niños de 6,5 a 11,4\% y en niñas de esa edad de 5,5 a 9,9\%, y de 12 a 17 años cifras similares. El sobrepeso aumentó hasta un $22 \%$.

Las complicaciones asociadas a la obesidad ${ }^{8-11}$ principalmente se desarrollan en la edad adulta, aunque cada vez se están viendo mas temprano en la edad pediátrica, como síndrome metabólico, diabetes tipo II, dislipemia, hipertensión arterial, arteriosclerosis, ferropenia, persistencia de la obesidad de adultos, sobre todo en los casos en los que el rebote adiposo típico de la niñez se adelanta antes de los 5 años, maduración puberal precoz, apnea nocturna, problemas por sobrecarga osteoarticular, aumento de mortalidad de adultos etc.
Debido al gran problema de salud pública que supone la obesidad, es muy importante poder hacer un diagnostico precoz en la infancia y prevenir y manejar la obesidad mejorando la función metabólica, disminuyendo así el riesgo de las complicaciones asociadas. La prevención ha de realizarse de forma universal a toda la población y selectiva a los sujetos con factores de riesgo. Las intervenciones principalmente son educacionales respecto a estilos de alimentación y de vida, fomentando una dieta equilibrada y ejercicio físico. Pueden realizarse tanto en el medio escolar, familiar o sanitario.

Dado el creciente aumento de la obesidad infantil en el primer mundo ${ }^{12}$, catalogado por algunos como epidemia, nos parece interesante conocer exactamente su prevalencia en la población pediátrica de nuestro medio de trabajo, así como la tendencia de la edad de inicio de la enfermedad, la evolución a lo largo de la edad pediátrica y si existen factores, como edad, sexo o hipertensión arterial, asociados a la obesidad/sobrepeso. De esta forma, podremos iniciar las medidas preventivas generales contra la obesidad en el momento más idóneo y durante el tiempo necesario. 


\section{MATERIAL Y MÉTODO}

Se ha realizado un estudio retrospectivo de seguimiento de una cohorte de todos los niños nacidos en 1992 en el área de Salud de Calzada de Calatrava $(n=92)$. Esta Zona Básica de Salud tiene una población pediátrica total de 0 a 14 años de unos 830 niños.

Las fuentes utilizadas son las historias clínicas del centro de salud, tanto en formato de papel como informático. En los casos en los que faltaba la revisión de los 14 años, se fue a realizar las mediciones al único instituto de enseñanza obligatoria de la zona hasta los 16 años.

Los datos recogidos de cada paciente fueron: peso, talla e IMC en la revisiones de recién nacido, 2 años, 6-7 años, 10-11 años y 14-15 años, y de tensión arterial de las dos últimas revisiones. Valoramos el IMC de cada paciente para ver si eran patológicos según los estándares para su edad y sexo por los dos sistemas de referencia ya comentados ${ }^{1,2}$, y la tensión arterial según las tablas para su edad, sexo y percentil de talla ${ }^{13}$.

De cada paciente obeso o con sobrepeso se calculó, a partir del momento de su debut, la evolución de la desviación estándar de la media de su IMC, para así tener una valoración cuantitativa exacta de la mejoría o no de su obesidad a lo largo del desarrollo de cada paciente.

Los datos fueron introducidos en una base de Excel, para después analizarlos con el programa estadístico SPSS.

\section{RESULTADOS}

La proporción, según el sexo, fue de 54,9\% hombres y $45,1 \%$ mujeres $(n=92)$. La prevalencia de obesidad y sobrepeso en las distintas edades de los controles del niño sano, como podemos ver en la tabla 1, ha experimentado un notable aumento, sobre todo desde los 6 a los 11 años, llegando hasta alrededor de un $35 \%$ en total, a partir de un $7 \%$ al nacimiento y un $10 \%$ a los 2 años, y estabilizándose después en un $30 \%$ a los 14 años.

Como podemos observar en la tabla 1, los valores de la prevalencia de obesidad y sobrepeso varían mucho según los estándares de referencia que usemos. Estos resultados se comprenden mejor analizando la figura 1: es mucho mas fácil ser diagnosticado de sobrepeso y menos de obeso con los estándares de Cole et al y al revés con los de Hernández y cols. A los 6 años los valores para diagnosticar sobrepeso y

\begin{tabular}{|c|c|c|c|c|c|}
\hline Edad & $\begin{array}{l}\text { PREVALENCIA } \\
\text { DE SOBREPESO }\end{array}$ & $\begin{array}{l}\text { INTERVALO DE CON- } \\
\text { FIANZA AL95\% }\end{array}$ & $\begin{array}{l}\text { PREVALENCIA DE } \\
\text { OBESIDAD }\end{array}$ & $\begin{array}{l}\text { INTERVALO DE CON- } \\
\text { FIANZA AL } 95 \%\end{array}$ & TOTAL \\
\hline \multicolumn{6}{|c|}{ SEGUN CRITERIOS DE COLE ET AL. } \\
\hline $\mathrm{RN}$ & No hay referencias & & No hay referencias & & \\
\hline $\begin{array}{l}2 \text { años } \\
6 \text { años } \\
11 \text { años } \\
14 \text { años }\end{array}$ & $\begin{array}{c}9,8 \% \\
25,6 \% \\
25,6 \% \\
22 \%\end{array}$ & $\begin{array}{l}3-17 \% \\
6-21 \% \\
3-24 \% \\
4-25 \%\end{array}$ & $\begin{array}{l}2,4 \% \\
6,1 \% \\
11 \% \\
9,8 \%\end{array}$ & $\begin{array}{l}11-32 \% \\
20-41 \% \\
21-42 \% \\
22-43 \%\end{array}$ & $\begin{array}{l}12.2 \% \\
31.7 \% \\
36.6 \% \\
31.8 \%\end{array}$ \\
\hline \multicolumn{6}{|c|}{ SEGUN CRITERIOS DE HERNANDEZ Y COLS. } \\
\hline $\begin{array}{l}\text { RN } \\
2 \text { años } \\
6 \text { años } \\
11 \text { años } \\
14 \text { años }\end{array}$ & $\begin{array}{c}7,3 \% \\
2,4 \% \\
9,8 \% \\
8,5 \% \\
13,4 \%\end{array}$ & $\begin{array}{l}3-12 \% \\
1-14 \% \\
3-12 \% \\
3-12 \% \\
8-13 \%\end{array}$ & $\begin{array}{c}0 \% \\
6,1 \% \\
6,1 \% \\
22 \% \\
18,3 \%\end{array}$ & $\begin{array}{c}0-4 \% \\
2-12 \% \\
2-11 \% \\
7-24 \% \\
6-14 \%\end{array}$ & $\begin{array}{c}7.3 \% \\
8.5 \% \\
15.9 \% \\
30.5 \% \\
31.7 \%\end{array}$ \\
\hline
\end{tabular}

Tabla 1. Prevalencia de obesidad en nuestra población a distintas edades considerando los datos perdidos, según criterios de Colé y de Hernández. RN: recién nacido.

\begin{tabular}{|c|c|c|c|c|c|}
\hline & IMC RN & $\begin{array}{c}\text { IMC A LOS } 2 \\
\text { AÑOS }\end{array}$ & $\begin{array}{c}\text { IMC A LOS } 6 \\
\text { AÑOS }\end{array}$ & $\begin{array}{l}\text { IMC A LOS } \\
11 \text { AÑOS }\end{array}$ & $\begin{array}{l}\text { IMC A LOS } 14 \\
\text { AÑOS }\end{array}$ \\
\hline $\begin{array}{l}\text { Nº casos válidos } \\
\text { Perdidos } \\
\text { Media } \\
\text { Mediana } \\
\text { Moda* } \\
\text { Desv.típ } \\
\text { Mínimo } \\
\text { Máximo }\end{array}$ & $\begin{array}{c}80 \\
2 \\
13.5604 \\
13.7750 \\
13.45 \\
1.1320 \\
10.80 \\
15.78\end{array}$ & $\begin{array}{c}80 \\
2 \\
16.4747 \\
16.2650 \\
15.32(\mathrm{a}) \\
1.4696 \\
13.63 \\
20.61\end{array}$ & $\begin{array}{c}80 \\
2 \\
16.7118 \\
16.0950 \\
15.15 \\
2.1366(\uparrow) \\
13.06 \\
23.05\end{array}$ & $\begin{array}{c}78 \\
4 \\
19.8051(\uparrow) \\
19 \\
14.38 \\
3.6180(\uparrow) \\
14.38 \\
28.36\end{array}$ & $\begin{array}{c}75 \\
7 \\
21.7911 \\
21.4200 \\
18.64(\mathrm{a}) \\
3.7901(\uparrow) \\
16.07 \\
30.85\end{array}$ \\
\hline
\end{tabular}

Tabla 2. Datos del IMC (Índice de Masa Corporal) de nuestra población a distintas edades. "Existen varias modas, se muestra el menor de los valores. 
obesidad son más bajos según Cole et al que según Hernández y cols.

Respecto a la alteración en la $1^{\circ}$ toma de tensión arterial durante la revisión de los 11 y 14 años, al analizar los pacientes obesos de 14 años diagnosticados por los criterios de Cole et al, hemos encontrado una asociación entre obesidad e hipertensión arterial, con un resultado estadísticamente significativo ( $p$ $=0.003$ ): 2 hipertensos de los 8 niños obesos, frente a 2 hipertensos de 67 niños no obesos (figura 2). No hemos encontrado relación significativa entre obesidad y otros factores analizados, como el sexo o la edad al debut.

También hemos calculado las curvas de IMC de nuestra población y las medias y las desviaciones típicas, que se encuentran en rangos similares a las publicadas por Hernández y cols, salvo a los 11 años donde la media y la desviación típica son superiores en nuestra población, y a los 14 años la desviación típica (tabla 2).

Por último, hemos calculado en cada paciente con obesidad o sobrepeso, en algún momento de nuestro estudio, el valor de la desviación estándar de la media en cada revisión posterior al inicio de su patología, para así ver la evolución exacta de su IMC respecto a su edad. Resumimos la evolución conjunta de nuestros pacientes en la figura 2, donde incluimos la media del sumatorio de las desviaciones estándar de los IMC de los obesos y con sobrepeso a cada edad, y como se puede comprobar hay un pico de máximo empeoramiento del IMC a los 11 años en nuestra población, y una tendencia ligera a mejorar a los 14 años los pacientes previamente diagnosticados, a pesar de que aumente la prevalencia de la obesidad en esas mismas edades.

\section{DISCUSIÓN}

La obesidad y el sobrepeso infantil se pueden considerar una pandemia de los últimos años, principalmente en los países desarrollados. Respecto a la prevalencia, en las publicaciones consultadas son similares a los datos obtenidos en nuestro estudio. También nosotros hemos podido observar un incremento en el número de casos en la población conforme han ido creciendo, desde alrededor de un $6 \%$ a los 6 años hasta un 18 $\%$ a los 14 años en esos mismos pacientes.

Respecto al sobrepeso, según criterios de Cole et al, al llegar a los 14 años alcanzamos en nuestra población un $22 \%$, al mismo nivel que las publicaciones recientes del estudio NHANES $\mathrm{III}^{7}$, realizado por el Center for Disease Control de Estados Unidos.

La condición de obesidad infantil, puede favorecer la presencia de otros factores de riesgo cardiovasculares como la hipertensión.

Por la similitud de nuestros resultados con las publicaciones consultadas, creemos que la obesidad y el sobrepeso constituyen un problema real e instaurado en nuestra población Española, que debemos considerar para intentar diagnosticarlos lo antes posible y prevenirlos muy activamente desde la consulta de atención primaria, pues como podemos comprobar es una epidemia en expansión que habría que intentar frenar.

\section{AGRADECIMIENTOS:}

A los profesores de educación física del Instituto de Bachillerato Eduardo Valencia, por ayudarnos a obtener los parámetros antropométricos de los pacientes que a los 14 años no habían acudido al centro de salud para realizarse la revisión médica.

A todo el personal del Centro de Salud de Calzada de Calatrava por el apoyo recibido durante la realización de este trabajo.

\section{BIBLIOGRAFÍA}

1. Cole TJ, Bellizzi MC, Flegal KM, Dietz WH. Establishing a Standard definition for child overweight and obesity workvide. Internacional survey. BMJ. 2000; 320:1240-1243.

2. Sobradillo B, Aguirre A, Aresti U, y cols. Curvas y tablas de crecimiento. Estudio longitudinal y tranversal. Fundación F. Orbegozo. En: Ergon, ed. Patrones de crecimiento y desarrollo en España. Atlas de gráficas y tablas. Madrid: Ergon; 2004. p. 145-168.

3. Cañete R, Gl Camps M. Obesidad. Pediatr Integral 2007; XI (6):531-543.

4. PAIDOS' 84. Estudio epidemiológico sobre nutrición y obesidad infantil. Madrid: Jomagar; 1985.

5. Serra Majem II, Ribas Barba I, Aranceta Bartina J, Pérez Rodrigo C, Ssaavedra Santana P, Peña Quintana I. Obesidad intantil y juvenil en España. Resultados del estudio enkid (1998-2000). Med Clin (Barc). 2003; 121(19):725-32.

6. González-Gross M, Ruiz JR, Moreno LA, De Rufino-Rivas P Garaulet M, Mesana MI, et al. AVENA Group. Body composition and physical performance of Spanish adolescents: the AVENA pilot study. Acta Diabetol. 2003; 40:299-301.

7. US Department of Health and Human Services.Centers for Disease Control and Prevention.National Center for Health Statistics.National Healthand United States. BMIAGE.

8. Reilly JJ, Methven E, McDowell ZC, Hacking B, Alexander D, Stewart L et al. Health consequences of obesity.. Arch Dis Child. 2003; 88:748-52.

9. Muñoz Calvo M.T. Síndrome metabólico. Pediatr integral 2007; XI(7):615-622.

10. Freedman DS, Mei Z, Srinivasan SR, Berenson GS, Dietz. $\mathrm{WH}$. Cardiovascular risk factors and excess adiposity among overweight children and adolescents: the Bogalusa Heart Study. J Pediatr. 2007; 150:12-7.

11. Grupo colaborativo español para el estudio de los factores de riesgo cardiovascular en la infancia y adolescencia. Factores de riesgo cardiovascular en la infancia y adolescencia en España. Estudio Ricardin II: valores de referencia. An Pediatr (Bar). 1995; 43:11-7.

12. Agence nationale d'accréditation et d'évaluation en santé (Anaes). Prise en charge de l'obésité de l'enfant et de l'adolescent; 2004.

13. The Fourth Report on the diagnosis, evaluación, and treatment of high blood pressure in children and adolescents. Pediatrics. 2004; 114:555-576. 